\title{
On a Question of Nearly Minimal Identification of Functions
}

\author{
Sanjay Jain \\ School of Computing \\ National University of Singapore \\ Singapore 119260 \\ sanjay@comp.nus.edu.sg
}

\begin{abstract}
Suppose $\mathcal{A}$ and $\mathcal{B}$ are classes of recursive functions. $\mathcal{A}$ is said to be an $m$-cover $(*$-cover) for $\mathcal{B}$, iff for each $g \in \mathcal{B}$, there exsits an $f \in \mathcal{A}$ such that $f$ differs from $g$ on at most $m$ inputs (finitely many inputs). $\mathcal{C}$, a class of recursive functions, is a-immune iff $\mathcal{C}$ is infinite and every recursively enumerable subclass of $\mathcal{C}$ has a finite $a$-cover. $\mathcal{C}$ is a-isolated iff $\mathcal{C}$ is finite or $a$-immune.

Chen [Che81] conjectured that every class of recursive functions that is $\mathbf{M E x}_{m}^{*}$-identifiable is $*$-isolated. We refute this conjecture.
\end{abstract}

\section{Introduction}

Formal definitions of notions informally discussed below are given in Section 3. Gold's [Gol67] criterion of identification of functions may be described as follows: A learning machine $\mathbf{M}$ is said to identify (or learn) a function $f$ just in case $\mathbf{M}$, when presented with the graph of $f$, outputs a sequence of programs that converges (in the limit) to a program for $f$. The above criterion of identification is called Ex-identification (Ex stands for explains). Freivalds [Fre75] (see also [Che81, Che82]) introduced the notion of nearly minimal identification, by placing an additional restriction on size of the final programs. In this criterion, the learning machine is required to converge to a program whose size is within a recursive factor of the size of the smallest program for the input function.

The above notions of identification can be extended in the following two directions:

- Error Bound ([BB75, CS83]): The above model may be relaxed by allowing the learning machine to converge to a program which may make some errors in computing the input function. An error bound of a natural number $m$ means that the final program makes at most $m$ errors in computing the input function. An error bound of $*$ means that the final program makes at most finitely many errors in computing the input function.

- Mind-Change Bound ([CS83, BF74]): The above model may be restricted by placing a bound on the number of mind changes allowed by the learning machine. A mind change bound of a natural number $m$ means that the learning machine may make at most $m$ mind changes before converging to the final program. A mind change bound of $*$ means that the learning machine may make at most finitely many mind changes before converging to the final program (note that $*$-mind change bound is equivalent to the Gold's notion of identification in the limit). 
Chen [Che81] showed that the recursively enumerable (r.e.) classes of functions that can be identified in the nearly minimal sense with $m$-errors and with a mind change bound of $n$ (where $m$ and $n$ are natural numbers) are not very complex - they can be "approximated" with at most $m$-errors using a finite class of functions. For a recursively enumerable classes, this latter notion of being approximated with at most $m$-errors, by a finite class of functions, is referred to as being $m$-isolated. Chen [Che81] also showed that classes of functions which can be nearly-minimallyidentified with $*$-errors, but with only 0 -mind changes, are $*$-isolated. The question of $*$-errors, but with mind change bound of $n>0$, was left open by Chen. He conjectured that such classes would also be $*$-isolated.

In this paper we refute Chen's conjecture. Thus complex r.e. classes can be identified in the nearly-minimal sense with $*$-errors and a nonzero mind change bound.

We now proceed formally.

\section{Notation}

Recursion-theoretic concepts not explained below are treated in [Rog67]. $N$ denotes the set of natural numbers, $\{0,1,2, \ldots\}$. All conventions regarding range of variables apply, with or without decorations $^{1}$, unless otherwise specified. The symbols $i, j, k, l, m, n, s, t, u, x, y$, and $z$, range over natural numbers unless otherwise specified. $\operatorname{card}(S)$ denotes the cardinality of a set $S$. * denotes a nonmember of $N$ and is assumed to satisfy $(\forall n \in N)[n<*<\infty]$. Thus, $\operatorname{card}(S) \leq *$ means that cardinality of the set $S$ is finite. $a$ and $b$ range over $N \cup\{*\}$. $\max (), \min ($ ) denote the maximum and minimum of a set, respectively. By convention $\max (\emptyset)=0$ and $\min (\emptyset)=\infty$.

$\mathcal{R}$ denotes the set of all total recursive functions. $\mathcal{A}, \mathcal{B}, \mathcal{C}$, and $\mathcal{S}$ range over subsets of $\mathcal{R}$. $h, f$, and $g$ range over total recursive functions. $\eta$ ranges over partial functions. domain $(\eta)$ denotes the domain of $\eta$. For $a \in N \cup\{*\}$, we say that $\eta_{1}={ }^{a} \eta_{2}$ (read: $\eta_{1}$ is an $a$-variant of $\eta_{2}$ ) iff $\operatorname{card}\left(\left\{x \mid \eta_{1}(x) \neq \eta_{2}(x)\right\}\right) \leq a$. Thus, $\eta_{1}={ }^{*} \eta_{2}$ means that $\eta_{1}$ and $\eta_{2}$ are finite variants of each other.

We let $\varphi$ denote a standard acceptable programming system. $\varphi_{i}$ denotes the partial recursive function computed by the $i^{\text {th }}$ program in the standard acceptable programming system $\varphi$. We often refer to the $i^{\text {th }}$ program as program $i . p$ ranges over total functions, with its range being interpreted as programs. For a recursive function $f, \operatorname{MinProg}(f)$ denotes the minimal program for $f$ (in the $\varphi$ system), i.e., $\operatorname{Min} \operatorname{Prog}(f)=\min \left(\left\{i \mid \varphi_{i}=f\right\}\right.$ ).

A class $\mathcal{S}$ of recursive functions is said to be recursively enumerable iff there exists a recursive set $Z$ such that $\mathcal{S}=\left\{\varphi_{i} \mid i \in Z\right\}$.

$\langle i, j\rangle$ stands for an arbitrary computable one to one encoding of all pairs of natural numbers onto $N$ [Rog67].

The quantifiers ' $\exists$ ', ' $\forall$ ', ' $\forall{ }^{\infty}$ ', and ' $\exists$ ' respectively denote 'there exists', 'for all', 'for all but finitely many', and 'there exist infinitely many'.

\section{Learning Paradigms}

For any partial function $\eta$ and any natural number $n$ such that, for each $x<n, \eta(x) \downarrow$, we let $\eta[n]$ denote the finite initial segment $\{(x, \eta(x)) \mid x<n\}$. Let SEQ $=\{f[n] \mid f \in \mathcal{R} \wedge n \in N\}$.

\footnotetext{
${ }^{1}$ Decorations are subscripts, superscripts, primes and the like.
} 
Definition 1 [Gol67] A learning machine is an algorithmic device which computes a mapping from SEQ into $N \cup\{$ ? $\}$ such that, if $\mathbf{M}(f[n]) \neq$ ?, then $\mathbf{M}(f[n+1]) \neq$ ?

We let $\mathbf{M}$, with or without decorations, range over learning machines. In Definition 1 above, '?' denotes the situation when $\mathbf{M}$ outputs "no conjecture" on some member of SEQ.

In Definition 2 below we spell out what it means for a learning machine to converge in the limit.

Definition 2 Suppose $\mathbf{M}$ is a learning machine and $f$ is a computable function. $\mathbf{M}(f) \downarrow$ (read: $\mathbf{M}(f)$ converges $)$ just in case $(\exists i)\left(\forall^{\infty} n\right)[\mathbf{M}(f[n])=i]$. If $\mathbf{M}(f) \downarrow$, then $\mathbf{M}(f)$ is defined $=$ the unique $i$ such that $\left(\forall^{\infty} n\right)[\mathbf{M}(f[n])=i]$, otherwise we say that $\mathbf{M}(f)$ diverges (written: $\left.\mathbf{M}(f) \uparrow\right)$.

\subsection{Explanatory Function Identification}

We now formally define the criteria of inference considered in this paper.

Definition 3 [Gol67, CS83, BB75, BF74] Suppose $a, b \in N \cup\{*\}$.

(1) A learning machine $\mathbf{M}$ is said to $\mathbf{E x}_{b}^{a}$-identify $f \in \mathcal{R}$ (written: $f \in \mathbf{E x}_{b}^{a}(\mathbf{M})$ ) just in case $\left(\exists i \mid \varphi_{i}={ }^{a} f\right)\left(\forall^{\infty} n\right)[\mathbf{M}(f[n])=i]$ and $\operatorname{card}(\{n \mid ? \neq \mathbf{M}(f[n]) \neq \mathbf{M}(f[n+1])\}) \leq b$.

(2) $\mathbf{E x}_{b}^{a}=\left\{\mathcal{C} \mid(\exists \mathbf{M})\left[\mathcal{C} \subseteq \mathbf{E x}_{b}^{a}(\mathbf{M})\right]\right\}$.

For a given $f$ and $\mathbf{M}$, we refer to each instance of the case, $? \neq \mathbf{M}(f[n]) \neq \mathbf{M}(f[n+1])$ as a mind change by $\mathbf{M}$ on $f$. Intuitively, in $\mathbf{E x}_{b}^{a}$, the superscript $a$ refers to the error bound on the final program, and subscript $b$ refers to the mind change bound. We often refer to $\mathbf{E x}_{*}^{a}$ as $\mathbf{E x}^{a}$, $\mathbf{E x}_{b}^{0}$ as $\mathbf{E x}_{b}$ and $\mathbf{E x}_{*}^{0}$ as $\mathbf{E x}$.

\subsection{Nearly Minimal Identification}

We next consider nearly minimal identification criteria.

Definition 4 [Fre75, Che82] Suppose $a, b \in N \cup\{*\}$.

(1) Suppose $h$ is a recursive function. A learning machine $\mathbf{M}$ is said to $h$-MEx $\mathbf{x}_{b}^{a}$-identify $f \in \mathcal{R}$ (written $f \in h$-MEx $\mathbf{x}_{b}^{a}(\mathbf{M})$ ) iff $\mathbf{M} \mathbf{E x}_{b}^{a}$-identifies $f$ and $\mathbf{M}(f) \leq h(\operatorname{MinProg}(f)$ ).

(2) $\mathbf{M E x}_{b}^{a}=\left\{\mathcal{C} \mid(\exists \mathbf{M})(\exists h \in \mathcal{R})\left[\mathcal{C} \subseteq h-\mathbf{M E x}_{b}^{a}(\mathbf{M})\right]\right\}$.

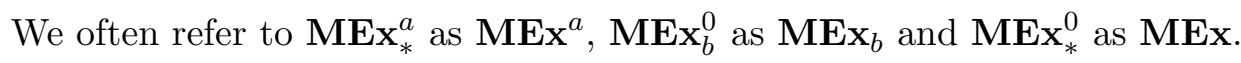

Theorem 5 [Che82, Fre75, Jai95] For all $m, n \in N, a \in N \cup\{*\}$.

(1) $\mathbf{E x}-\mathbf{M E x}^{m} \neq \emptyset$.

(2) $\mathbf{E x}_{0}^{0}-\mathbf{M E x}_{n}^{*} \neq \emptyset$.

(3) $\mathbf{E x}_{n}^{a} \subseteq \mathbf{M E x}^{a}$.

(4) $\mathbf{E x}^{*}=\mathbf{M E x}$.

(5) $\mathbf{M E x}_{n+1}^{0}-\mathbf{E x}_{n}^{*} \neq \emptyset$.

(6) $\mathbf{M E x}_{0}^{m+1}-\mathbf{E x}^{m} \neq \emptyset$. 


\subsection{Isolated Classes}

Definition 6 [Che81] Suppose $\mathcal{A}$ and $\mathcal{B}$ are classes of recursive functions. $\mathcal{B}$ is an a-cover of $\mathcal{A}$ iff for each $g \in \mathcal{B}$, there exists an $f \in \mathcal{A}$, such that $f={ }^{a} g$.

Definition 7 [Che81, $\operatorname{Rog} 67] \mathcal{C}$ is a-immune iff (a) $\mathcal{C}$ is infinite and (b) every recursively enumerable subclass of $\mathcal{C}$ has a finite $a$-cover.

Definition 8 [Che81] $\mathcal{C}$ is a-isolated iff $\mathcal{C}$ is finite or $\mathcal{C}$ is $a$-immune.

Chen [Che81] established the following two results.

Theorem 9 [Che81] Suppose $m, n \in N$, and $\mathcal{S} \in \mathbf{M E x}_{n}^{m}$. Then $\mathcal{S}$ is $m$-isolated.

Theorem 10 [Che81] Suppose $\mathcal{S} \in \mathbf{M E x}_{0}^{*}$. Then $\mathcal{S}$ is *-isolated.

Based on above results, Chen conjectured that, for $n \in N$, every $\mathcal{S} \in \mathbf{M E x}_{n}^{*}$ is $*$-isolated. We surprisingly refute his conjecture.

\section{Main Theorem}

Theorem 11 There exists an infinite recursively enumerable class $\mathcal{S} \in \mathbf{M E x}_{1}^{*}$ such that $\mathcal{S}$ is not *-isolated.

Proof. Let

$\mathcal{C}_{1}=\left\{f \mid \varphi_{f(\langle 0,0\rangle)}={ }^{*} f \wedge f(\langle 0,0\rangle) \leq \operatorname{MinProg}(f) \wedge(\forall x)[f(\langle 1, x\rangle)=0]\right\}$,

$\mathcal{C}_{2}=\left\{f \mid\left(\forall^{\infty} x\right)[f(x)=0] \wedge(\exists x)[f(\langle 1, x\rangle) \neq 0]\right\}$,

and $\mathcal{C}=\mathcal{C}_{1} \cup \mathcal{C}_{2}$.

Intuitively, $\mathcal{C}_{1}$ is a class of (nearly) self-describing functions, where a small program for a finite variant of the function is coded into the function itself. $\mathcal{C}_{2}$ is a subclass of almost everywhere 0 functions. Additionally, we code into the functions (using $\{\langle 1, x\rangle \mid x \in N\}$ ) whether it is from $\mathcal{C}_{1}$ or $\mathcal{C}_{2}$.

It is easy to verify that $\mathcal{C}$ is in $\mathbf{M E x}_{1}^{*}$. We will construct the required $\mathcal{S}$ as an appropriate recursively enumerable subset of $\mathcal{C}$. Intuitively, the idea is to use an appropriate subclass of $\mathcal{C}_{1}$ to ensure that $\mathcal{S}$ is $*$-isolated. $\mathcal{C}_{2}$ is added to this subclass, to ensure that $\mathcal{S}$ is recursively enumerable. We now continue with the formal construction of $\mathcal{S}$.

Using Operator Recursion Theorem [Cas74] we will define a recursive, 1-1, increasing function $p$ such that the functions $\varphi_{p(i)}$ satisfy the following four properties:

(A) For all $x, \varphi_{p(i)}(\langle 0, x\rangle)=p(i)$;

(B) For all $x, \varphi_{p(i)}(\langle 1, x\rangle)=0$;

(C) $\varphi_{p(i)}$ is undefined on exactly one input; let this input be called $u_{i}$;

(D) For all $j<p(i)$, either $\varphi_{j}$ is non-total, or there exists an $x<u_{i}$ such that $\varphi_{j}(x) \neq \varphi_{p(i)}(x)$. Let $f_{i}$ be defined as follows:

$$
f_{i}(x)= \begin{cases}\varphi_{p(i)}(x), & \text { if } x \neq u_{i} \\ 0, & \text { otherwise }\end{cases}
$$

Let $\mathcal{S}=\left\{f_{i} \mid i \in N\right\} \cup \mathcal{C}_{2}$ 
It is easy to verify that $\mathcal{S}$ is recursively enumerable. Moreover, by property (A) $\mathcal{S}$ is not $*-$ isolated. It is also easy to verify (using properties $(\mathrm{A})-(\mathrm{D})$ ) that $f_{i} \in \mathcal{C}_{1}$. Thus, $\mathcal{S} \subseteq \mathcal{C}$ and $\mathcal{S} \in \mathrm{MEx}_{1}^{*}$.

We now give the construction of $\varphi_{p(i)}$ satisfying the properties (A) to (D) above. By operator recursion theorem [Cas74] there exists a $1-1$, recursive, increasing function $p$ such that $\varphi_{p(i)}$ may be defined in stages as follows.

Let $u_{i}^{0}=\min (N-\{y, x\} \mid y \in\{0,1\} \wedge x \in N)$. Let Cancel $_{i}^{0}=\emptyset$. Intuitively, $u_{i}^{s}$ denotes the intended value of $u_{i}$ as at the beginning of stage $s$. Cancel $_{i}^{s}$ is used to keep track of programs $<p(i)$, against which $\varphi_{p(i)}$ has diagonalized against before stage $s$. Go to stage 0 .

Stage $s$

1. Dovetail steps 2 and 3 until step 2 succeeds. If and when step 2 succeeds, go to step 4 .

2. Search for a $j<p(i)$, such that $j \notin$ Cancel $_{i}^{s}$, and $\varphi_{j}\left(u_{i}^{s}\right) \downarrow$.

3. For $z=0$ to $\infty$ Do

If $z \neq u_{i}^{s}$ and $\varphi_{p(i)}(z)$ has not been defined upto now, Then

Let $\varphi_{p(i)}(z)=p(i)$, if $z=\langle 0, x\rangle$ for some $x \in N$;

Let $\varphi_{p(i)}(z)=0$, if $z=\langle y, x\rangle$ for some $x \in N$ and $y \neq 0$;

EndFor

4. If and when step 2 succeeds, then let $j$ be as in step 2 .

Let Cancel $_{i}^{s+1}=$ Cancel $_{i}^{s} \cup\{j\}$.

Let $\varphi_{p(i)}\left(u_{i}^{s}\right)=\varphi_{j}\left(u_{i}^{s}\right)+1$.

Let $u_{i}^{s+1}$ be the minimum number $z$ such that $\varphi_{p(i)}(z)$ has not been defined upto now, and $z \notin\{\langle y, x\rangle \mid y \in\{0,1\} \wedge x \in N\}$.

Go to stage $s+1$.

End Stage $s$

We now argue that $\varphi_{p(i)}$ defined above satisfies properties (A) to (D) above. First note that there are only finitely many stages. This is so since each time a new stage $>0$ is entered, step 4 in the previous stage must have diagonalized against a new program $j<p(i)$. Since there are at most finitely many programs less than $p(i)$, there are at most finitely many stages that are executed. Let $s$ be the last stage that is entered but never finished. Let $u_{i}=u_{i}^{s}$ and Cancel $_{i}=$ Cancel $_{i}^{s}$. It is now easy to verify that (A), (B) and (C) are satisfied. Also, for all $j<p(i)$, either $j \in$ Cancel $_{i}$, or $\varphi_{j}\left(u_{i}\right) \uparrow$. In case $j \in$ Cancel $_{i}$, then by step 4 of the construction, there exists a $z<u_{i}$ such that $\varphi_{j}(z) \downarrow \neq \varphi_{p(i)}(z) \downarrow$. Thus, (D) is satisfied. This completes the proof of the theorem.

Corollary 12 For all $n>0$, there exists a recursively enumerable class $\mathcal{S} \in \mathbf{M E x}_{n}^{*}$ such that $\mathcal{S}$ is not $*$-isolated.

\section{Acknowledgements}

We thank an anonymous referee for several helpful suggestions which improved the presentation of the paper. Specially acknowledged are his suggestions for improving the Introduction section to make it accessible to a wider audience. 


\section{References}

[BB75] L. Blum and M. Blum. Toward a mathematical theory of inductive inference. Information and Control, 28:125-155, 1975.

[BF74] J. Bārzdiņš and R. Freivalds. Prediction and limiting synthesis of recursively enumerable classes of functions. Latvijas Valsts Univ. Zimatm. Raksti, 210:101-111, 1974.

[Cas74] J. Case. Periodicity in generations of automata. Mathematical Systems Theory, 8:15-32, 1974.

[Che81] K. J. Chen. Tradeoffs in Machine Inductive Inference. PhD thesis, SUNY/Buffalo, 1981.

[Che82] K. J. Chen. Tradeoffs in inductive inference of nearly minimal sized programs. Information and Control, 52:68-86, 1982.

[CS83] J. Case and C. Smith. Comparison of identification criteria for machine inductive inference. Theoretical Computer Science, 25:193-220, 1983.

[Fre75] R. Freivalds. Minimal Gödel numbers and their identification in the limit. In Mathematical Foundations of Computer Science, volume 32 of Lecture Notes in Computer Science, pages 219-225. Springer-Verlag, 1975.

[Gol67] E. M. Gold. Language identification in the limit. Information and Control, 10:447-474, 1967.

[Jai95] S. Jain. On a question about learning nearly minimal programs. Information Processing Letters, 53(1):1-4, 1995.

[Rog67] H. Rogers. Theory of Recursive Functions and Effective Computability. McGraw-Hill, 1967. Reprinted by MIT Press in 1987. 